\title{
Increased aortic stiffness and blood pressure in non-classic Pompe disease
}

\author{
Stephan C. A. Wens • Esther Kuperus • Francesco U. S. Mattace-Raso • \\ Michelle E. Kruijshaar • Esther Brusse • Kees C. A. G. M. van Montfort • \\ Marjan Scheltens- de Boer • Eric J. G. Sijbrands • Ans T. van der Ploeg • \\ Pieter A. van Doorn
}

Received: 10 September 2013 /Revised: 19 November 2013 / Accepted: 22 November 2013 /Published online: 10 January 2014

(C) The Author(s) 2013. This article is published with open access at Springerlink.com

\begin{abstract}
Vascular abnormalities and glycogen accumulation in vascular smooth muscle fibres have been described in Pompe disease. Using carotid-femoral pulse wave velocity (cfPWV), the gold standard methodology for determining aortic stiffness, we studied whether aortic stiffness is increased in patients with Pompe disease. Eighty-four adult Pompe patients and 179 age- and gender-matched volunteers participated in this cross-sectional case-controlled study. Intima media thickness and the distensibility of the right common carotid artery were measured using a Duplex scanner. Aortic augmentation index, central pulse pressure, aortic reflexion
\end{abstract}

Communicated by: Olaf Bodamer

Stephan C. A. Wens and Esther Kuperus Equal contributors

S. C. A. Wens $\cdot$ E. Kuperus $\cdot$ E. Brusse $\cdot$ M. S.-. de Boer $\cdot$

P. A. van Doorn $(\square)$

Department of Neurology, Erasmus University Medical Center,

Mailbox 2040, 3000 CA Rotterdam, The Netherlands

e-mail: p.a.vandoorn@erasmusmc.nl

S. C. A. Wens • E. Kuperus • M. E. Kruijshaar • E. Brusse •

A. T. van der Ploeg • P. A. van Doorn

Center for Lysosomal and Metabolic Diseases, Erasmus MC,

Rotterdam, The Netherlands

F. U. S. Mattace-Raso • E. J. G. Sijbrands

Department of Internal Medicine, Erasmus MC, Rotterdam, The Netherlands

A. T. van der Ploeg

Department of Pediatrics, Division of Metabolic Diseases and

Genetics, Erasmus MC-Sophia, Rotterdam, The Netherlands

K. C. A. G. M. van Montfort

Department of Epidemiology and Biostatistics, Erasmus MC,

Rotterdam, The Netherlands time and cfPWV were assessed using the SphygmoCor ${ }^{\circledR}$ system. CfPWV was higher in patients than in volunteers ( 8.8 versus $7.4 \mathrm{~m} / \mathrm{s}, p<0.001$ ). This difference was still present after adjustment for age, gender, mean arterial blood pressure (MAP), heart rate and diabetes mellitus $(p=0.001)$, and was shown by subgroup analysis to apply to the 40 59 years age group $(p=0.004)$ and $60+$ years age group $(p=$ $0.01)$, but not to younger age groups $(p=0.99)$. Except for a shorter aortic reflexion time $(p=0.02)$, indirect indicators of arterial stiffness did not differ between patients and volunteers. Relative to volunteers (20\%), more Pompe patients had a history of hypertension (36 \%,p=0.005), and the MAP was higher than in volunteers (100 versus $92 \mathrm{mmHg}, p<0.001)$. This study shows that patients with non-classic Pompe disease have increased aortic stiffness and blood pressure. Whether this is due to glycogen accumulation requires further investigation. To reduce the potential risk of cardiovascular diseases, we recommend that blood pressure and other common cardiovascular risk factors are monitored regularly.

$\begin{array}{ll}\text { Abbreviations } \\ \text { AIx } & \text { augmentation index } \\ \text { ATr } & \text { aortic reflexion time } \\ \text { CfPWV } & \text { carotid-femoral pulse wave velocity } \\ \text { DBP } & \text { diastolic blood pressure } \\ \text { ERT } & \text { enzyme replacement therapy } \\ \text { IMT } & \text { intima media thickness } \\ \text { MAP } & \text { mean arterial blood pressure } \\ \text { PAS } & \text { periodic acid-Schiff } \\ \text { PPc } & \text { central pulse pressure } \\ \text { PWA } & \text { pulse wave analysis } \\ \text { SBP } & \text { systolic blood pressure }\end{array}$




\section{Introduction}

Pompe disease (OMIM 232300: acid maltase deficiency or glycogen storage disease type II) is an inheritable lysosomal storage disorder caused by a deficiency of acid $\alpha$-glucosidase that leads to glycogen accumulation in various body tissues, predominantly skeletal, cardiac and smooth muscle (Hirschhorn 2001; van der Ploeg and Reuser 2008). Patients with the classic-infantile form of the disease develop generalized hypotonia and a hypertrophic cardiomyopathy. Without treatment, these patients die in the first year of life due to cardiorespiratory failure (van den Hout et al 2003). In the nonclassic form of the disease, which can present at any age, progressive muscle weakness is the predominant symptom. In general, there is no significant cardiac involvement (Soliman et al 2008; van der Beek et al 2012).

Over the last two decades, several reports have hypothesized that glycogen accumulation in the smooth muscle tissue of arteries leads to vascular abnormalities in non-classic Pompe disease, such as cerebral aneurysms, basilar artery dolichoectasia, carotid dissection and dilated arteriopathy of the thoracic aorta (Braunsdorf 1987; Winkel et al 2003, 2005; Laforet et al 2008; Sacconi et al 2010; El-Gharbawy et al 2011). The first indication of increased aortic stiffness in nonclassic Pompe disease was reported in a study on 17 Pompe patients that used transthoracic Doppler echocardiography, a non-invasive tool that measures aortic stiffness indirectly (Nemes et al 2007). The presence of increased aortic stiffness may be relevant, since it is considered to be an independent risk factor for cardiovascular disease and mortality (MattaceRaso et al 2006; Verbeke et al 2011). The emerging gold standard for measuring it directly and non-invasively is tonometry of the carotid-femoral pulse wave velocity (cfPWV) (Van Bortel et al 2012).

To determine whether increased arterial stiffness is a feature of non-classic Pompe disease, we used various techniques including tonometry of the cfPWV to investigate the structural and functional vascular properties of different vascular territories in a large group of patients with non-classic Pompe disease. We compared the results with those in age- and gender-matched volunteers.

\section{Materials and methods}

\section{Study population}

Between March 2012 and June 2013 we performed a crosssectional single-centre case-controlled study in 84 patients with non-classic Pompe disease and 179 volunteers. All patients were 18 years or older. As the volunteers were matched for gender and age category, there were, per patient, at least two volunteers of the same gender and the same age category.
The volunteers were either the patients' partners, or were employees or students of Erasmus University Medical Center, or otherwise visitors to it. Informed consent was obtained from all participants. The study protocol was approved by the Medical Ethical Committee at Erasmus University Medical Center

\section{Cardiovascular risk factors}

All participants filled out a questionnaire to assess cardiovascular risk factors, which included gender, date of birth, length, weight, medication use, and comorbidities such as hypertension, diabetes mellitus, hypercholesterolemia and cardiovascular disease (i.e. transient ischemic attack, stroke, myocardial infarction or rhythm disturbances). Body mass index (BMI), which was calculated as body weight divided by height squared, was expressed as $\mathrm{kg} / \mathrm{m}^{2}$. An automatic mercury cuff sphygmomanometer on the right arm was used to measure heart rate and blood pressure, including the mean arterial blood pressure (MAP), when the patient was in supine position after $5 \mathrm{~min}$ of rest in a quiet and heat-controlled room. Hypertension was defined as a systolic blood pressure (SBP) $>$ $140 \mathrm{mmHg}$ and/or diastolic blood pressure (DBP) $>90 \mathrm{mmHg}$ (Mancia et al 2013).

\section{Vascular measurements}

Aortic stiffness was measured using arterial tonometry from the right radial, right carotid and right femoral arteries using the SphygmoCor ${ }^{\circledR}$ device (Sphygmocor version 7.1, AtCor Medical, Sydney, Australia) according to previously described procedures (van Dijk et al 2013). Transit distance was assessed on the basis of body surface measurement from the carotid artery to the femoral artery: $80 \%$ of this distance was used as pulse-wave-travelled distance (Van Bortel et al 2012). The primary outcome measure, cfPWV, was expressed in meters per second $(\mathrm{m} / \mathrm{s})$. The secondary and indirect outcome parameters for arterial stiffness measured with pulse wave analysis (PWA) of the right radial artery were central pulse pressure $(\mathrm{PPc})$, augmentation index (AIx) and aortic reflexion time (ATr). PPc was calculated as the difference between aortic systolic and diastolic pressure; AIx was calculated from the aortic pressure waveform obtained by applying a general transfer function to the radial pressure waveform. For the comparison between two groups, AIx was normalized to a heart rate of 75 beats per minute. ATr was calculated as the time interval between the foot of the pressure wave and the shoulder of the reflected wave (Laurent et al 2006; DeLoach and Townsend 2008; Huijben et al 2012).

The distensibility and IMT of the right common carotid artery were measured two centimetres below the carotid bifurcation using a vessel-wall movement-detector system (Art.Lab, Esaote Europe, Maastricht, the Netherlands) 
according to previously described procedures (van Dijk et al 2013). The distensibility coefficient, a local measurement of carotid stiffness, was calculated as $(2 \Delta \mathrm{D} / \mathrm{D}) / \mathrm{PP}\left(10^{-3} / \mathrm{kPa}\right)$ (Hoeks et al 1992), in which $\mathrm{D}$ is the diameter during diastole, $\Delta \mathrm{D}$ is the measured distensibility (systolic diameter - diastolic diameter), and PP is the aortic pulse pressure derived from radial applanation. Tonometry and ultrasonography were performed by two trained physicians (SW or EK).

\section{Statistical analyses}

Unless otherwise specified, data are presented as medians with interquartile ranges (IQR). Because cfPWV, PPc, AIx, ATr, distensibility and IMT were not normally distributed, the Mann-Whitney test was used to compare these outcomes between the total groups of patients and volunteers.

Linear regression analyses were used to study cfPWV separately. First, univariate regression analyses were performed. Next, the regression coefficients with a p-value less than 0.05 or variables that were considered clinically relevant were analysed in a multivariate regression model using bootstrap estimation. The final multivariate model contained age, gender, MAP, heart rate and diabetes mellitus.

We also performed a stratified analysis between patients and volunteers in three age groups: 20-39, 40-59 and 60+. To determine whether cfPWV was influenced by disease duration, treatment with ERT, and wheelchair or ventilator dependency, we also analysed cfPWV in separate multivariate regression models using bootstrap estimation. The final model for these analyses included four variables: age, gender, MAP and heart rate. The data were analysed using SPSS 20. A pvalue of less than 0.05 was considered statistically significant.

\section{Results}

\section{Study population}

Table 1 shows the characteristics of the two groups. In total, 84 patients with non-classic Pompe disease and 179 volunteers were included in the study. Twenty-six percent of the patients were wheelchair dependent, and $31 \%$ needed artificial ventilation. Eighty-two percent of the patients were treated with ERT and the median duration of treatment was 5 years (IQR 4-5). CfPWV could not be measured in nine patients, either because they could not lie in supine position due to respiratory failure, or because their use of accessory muscles made it difficult to visualize the carotid artery. Neither could cfPWV be measured in the other eight volunteers due to temporary technical problems with the SphygmoCor ${ }^{\circledR}$ system.
Table 1 Demographic and clinical characteristics

\begin{tabular}{lll}
\hline & Pompe patients & Volunteers \\
\hline Number of individuals & 84 & 179 \\
Age in years & $54(42-63)$ & $54(41-61)$ \\
Gender - male (\%) & $41(49)$ & $85(48)$ \\
Body mass index in kg/m & $24.7(22.0-27.3)$ & $24.0(22.2-26.0)$ \\
Smoking status (\%) & & \\
- Never & $36(43)$ & $107(60)$ \\
- Past & $41(49)$ & $53(29)$ \\
- Current & $7(8)$ & $19(11)$ \\
Medical history & & \\
- Hypertension (\%) & $30(36)^{\mathrm{a}}$ & $35(20)$ \\
- Diabetes mellitus (\%) & $5(6)$ & $6(3)$ \\
- Hypercholesterolemia (\%) & $8(10)$ & $18(10)$ \\
- Cardiovascular disease (\%) & $7(8)$ & $9(5)$ \\
Antihypertensive medication (\%) & $23(77)$ & $31(89)$ \\
Disease duration in years & $16(12-23)$ & - \\
Patients receiving ERT (\%) & $69(82)$ & - \\
Duration of ERT in years & $5(4-5)$ & - \\
Wheelchair use (\%) & $22(26)$ & $0(0)$ \\
Ventilator use (\%) & $26(31)$ & $0(0)$ \\
\hline
\end{tabular}

Continuous variables are given as medians with interquartile ranges (IQR), and categorical variables as numbers and percentages

${ }^{\mathrm{a}}$ In the past, more patients with Pompe disease than volunteers were diagnosed with hypertension ( $\mathrm{p}-0.005)$

\section{Cardiovascular risk factors}

In the past, more patients with Pompe disease than volunteers had been diagnosed with hypertension ( $p=0.005$, Table 1$)$. Seventy-seven per cent of the Pompe patients with an earlier diagnosis of hypertension used antihypertensive medication at the time of evaluation, and $89 \%$ in the group of volunteers. Despite treatment with antihypertensive medication, blood pressure continued to be high in $52 \%$ of the Pompe patients who were treated for hypertension and in $65 \%$ of the volunteers. As Table 2 shows, MAP was higher in patients than in volunteers (100 mmHg (IQR 90-111) versus $92 \mathrm{mmHg}$ (IQR 84-103), $p<0.001)$. Thirty-nine per cent of the patients met the criteria for hypertension (SBP $>140 \mathrm{mmHg}$ and/or DBP $>$ $90 \mathrm{mmHg}$ ), versus $20 \%$ of the volunteers $(p=0.001)$. At group level, patients with Pompe disease had a higher heart rate (75 beats per minute (bpm) (IQR 68-83)) than the volunteers (65 bpm (IQR 59-71), $p<0.001$ ).

There were no differences in the occurrence of comorbidities such as diabetes mellitus or hypercholesterolemia. Seven patients with Pompe disease had a medical history of cardiovascular disease: four of them (three female) had had a transient ischemic attack between the ages of 48 and 62 years); two male patients had developed atrial fibrillation at the ages of 37 and 64 years; and one female patient had had a 
Table 2 Functional and structural hemodynamic parameters in patients and volunteers

\begin{tabular}{llll}
\hline & Pompe patients $(n=84)$ & Volunteers $(n=179)$ & $P$-value \\
\hline Mean arterial pressure $(\mathrm{mmHg})$ & $100(90-111)$ & $92(84-103)$ & $<0.001$ \\
Heart rate (beats per minute) & $75(68-83)$ & $65(59-71)$ & $<0.001$ \\
Diameter carotid artery $(\mathrm{mm})$ & $6.6(6.1-7.3)$ & $7.1(6.5-7.8)$ & $<0.001$ \\
Intima media thickness $(\mu \mathrm{m})$ & $582(514-683)$ & $592(506-695)$ & 0.68 \\
Distensibility coefficient $\left(10^{-3} / \mathrm{kPa}\right)$ & $2.9(1.9-4.1)$ & $2.7(2.2-4.1)$ & 0.72 \\
Carotid-femoral pulse wave velocity $(\mathrm{m} / \mathrm{s})$ & $8.8(7.1-10.7)$ & $3.4(6.6-8.6)$ & $<0.001$ \\
Central pulse pressure $(\mathrm{mmHg})$ & $38(31-47)$ & $19.4(8.5-29.4)$ & 0.30 \\
Augmentation index $(\%)$ & $21.7(13.2-30.3)$ & $144(135-159)$ & 0.23 \\
Aortic reflexion time $(\mathrm{ms})$ & $140(132-149)$ & 0.02 \\
\hline
\end{tabular}

Continuous variables are given as medians with interquartile ranges (IQR). The diameter of the carotid artery was measured in 179 volunteers and 84 patients; IMT was measured in 179 volunteers and 83 patients; the distensibility coefficient was measured in 176 volunteers and 80 patients; cfPWV was measured in 171 volunteers and 79 patients; and PPc, Aix and ATr were measured in 178 volunteers and 83 patients

myocardial infarction at the age of 74 years. Nine volunteers had a medical history of cardiovascular disease. This was not statistically different from the seven Pompe patients $(p=0.30)$.

Aortic stiffness

As shown in Table 2, median cfPWV was $8.8 \mathrm{~m} / \mathrm{s}$ in patients with Pompe disease (IQR 7.1-10.7) and $7.4 \mathrm{~m} / \mathrm{s}$ in volunteers (IQR 6.6-8.6) $(p<0.001)$. This difference was still present after adjustment for age, gender, MAP, heart rate and diabetes mellitus $(p=0.001)$. Subgroup analysis showed that while it occurred in the $40-59$ years age group $(8.6 \mathrm{~m} / \mathrm{s}$ in patients versus $7.6 \mathrm{~m} / \mathrm{s}$ in volunteers, $p=0.004)$ and the $60+$ years (11.5 versus $9.3 \mathrm{~m} / \mathrm{s}, p=0.01$ ), it did not occur in the 20 39 years age group (Fig. 1). PPc and AIx did not differ significantly between patients and volunteers, while ATr was shorter in patients than in volunteers $(p=0.02)$.

CfPWV did not differ between Pompe patients whose disease duration was shorter than 15 years $(n=35)$ and those whose disease duration was longer than 15 years $(n=44)(9.1$ versus $9.4 \mathrm{~m} / \mathrm{s}, p=0.51)$. Neither did it differ between patients treated with ERT $(n=64)$ and treatment-naïve patients $(n=15)$ (9.4 versus $8.6 \mathrm{~m} / \mathrm{s}, p=0.17$ ). Similarly, cfPWV did not differ between wheelchair-dependent patients $(n=12)$ and ambulant patients $(n=62)(9.4$ versus $9.2 \mathrm{~m} / \mathrm{s}, p=0.75)$, or between ventilator-dependent patients $(n=18)$ and non-ventilated patients $(n=56)(9.6$ versus $9.1 \mathrm{~m} / \mathrm{s}, p=0.42)$.

\section{Carotid stiffness and IMT}

Table 2 shows that there were no significant differences in distensibility or IMT between patients and volunteers. The diameter of the carotid artery was smaller in patients than in volunteers (6.6 mm (IQR 6.1-7.3) versus 7.1 mm (IQR 6.5$7.8), p<0.001)$. Subgroup analysis showed that the distensibility was lower in patients with Pompe disease aged between
20 and 39 years than in volunteers $(p=0.03)$, but not in the other age-related subgroups.

Glycogen accumulation in vascular smooth muscle

Figure 2 shows the muscle biopsy of a 50-year-old male patient with Pompe disease who participated in this study and had no medical history of cardiovascular diseases. He developed the first symptoms at age 44, and now has severe limb-girdle weakness and decreased pulmonary function in sitting and supine positions. The biopsy was stained with periodic acid-Schiff (PAS) and shows glycogen storage in skeletal muscle fibres and in vascular smooth muscle fibres.

\section{Discussion}

We found that patients with the non-classic form of Pompe disease have increased aortic stiffness and blood pressure. These findings indicate clinical relevant involvement of the cardiovascular musculature in this group of Pompe patients.

Glycogen accumulation in smooth muscle fibres and in the endothelial layer of arteries has been shown in morphological studies and autopsy reports of patients with Pompe disease (Winkel et al 2003; Thurberg et al 2006; Kobayashi et al 2010). Figure 2 shows glycogen storage in vascular smooth muscle fibres in one of our adult patients with Pompe disease before the start of ERT. While it is likely that the increased glycogen storage in smooth muscle fibres results in an increased arterial stiffness, it is also possible that glycogen accumulation in the endothelium damages the vascular wall, making it more vulnerable to atherosclerosis; in the presence of other cardiovascular risk factors, this process might increase arterial stiffness. As vascular stiffness has also been described as depending on the distribution of collagen and elastin, an increase in abnormal collagen and a decrease in 
Fig. 1 Mean values of cfPWV in patients and volunteers per age category. The boxplots represent the mean values and $95 \% \mathrm{CI}$ of cfPWV per age category after adjustment for age, gender, mean arterial blood pressure, heart rate and diabetes mellitus. The number of patients and volunteers per age group are given in the bars

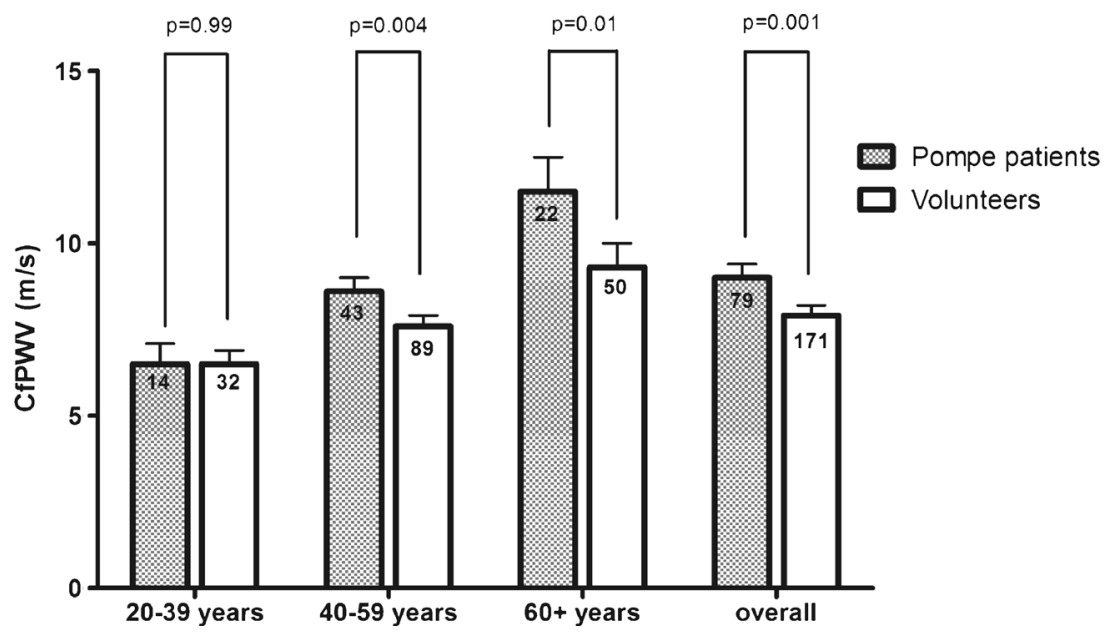

normal elastin contributes to greater arterial stiffness (Laurent et al 2006). During the course of the disease, patients with Pompe disease may develop striated muscle atrophy with some increase in connective tissue. If this process also occurred in the vascular smooth muscle fibres of Pompe patients, it would help to explain increased aortic stiffness.

Increased aortic stiffness has also been described in other lysosomal storage disorders such as Fabry disease and mucopolysaccharidosis type I (MPS I or Scheie syndrome). In Fabry disease, storage of glycosphingolipids takes place in vascular endothelial and smooth muscle cells, and in MPS I, accumulation of glycosaminoglycans in the connective tissue of the aortic wall impairs the formation of elastin and leads to changes in collagen formation (Nemes et al 2008; Collin et al 2012). It has been shown in Fabry disease that ERT can normalize aortic stiffness (Collin et al 2012). As most of the patients with Pompe disease enrolled in our cross-sectional study were treated with ERT, the difference in cfPWV between patients and volunteers might have been even more pronounced if it had been measured before the start of ERT. It would thus be interesting to establish whether aortic stiffness in Pompe patients decreases during ERT, and whether cfPWV can be used as a non-invasive tissue biomarker for disease progression and therapy response.

To the best of our knowledge, this is the first study to show that arterial blood pressure is increased in patients with Pompe disease. It is reasonable to assume that any structural changes in the blood vessels caused by the increased aortic stiffness would lead to higher blood pressure. Increased aortic stiffness and hypertension are both independent risk factors for cardiovascular disease and mortality (Mattace-Raso et al 2006; Verbeke et al 2011). It has been shown that patients with non-classic Pompe disease have improved survival when treated with ERT (Güngör et al 2013). Since cardiovascular diseases are more common at a higher age, Pompe patients will be more likely to develop cardiovascular diseases, as they owe their higher age to ERT. In these patients it is therefore important to closely monitor risk factors for cardiovascular diseases such as hypertension, hypercholesterolemia, diabetes mellitus and smoking, and to treat them when indicated. Although exercise and training, dietary changes and pharmacological treatments have sometimes been shown to reduce arterial stiffness in healthy individuals and other patient groups (Kingwell et al 1997; Girerd et al 1998; Balkestein et al 1999), it has not yet been investigated whether they are also beneficial in Pompe patients.

Although we expected Pompe disease to have a similar effect on the vascular structure of all arteries, and thereby to increase cfPWV and reduce distensibility, we found changes in carotid distensibility only in patients aged between 20 and 39 years. In those with high blood pressure and diabetes it has been reported that the aorta stiffened more than the carotid
Fig. 2 Glycogen accumulation in vascular smooth muscle fibres. Muscle biopsies of adult patients with non-classic Pompe disease stained with periodic acid-Schiff (PAS) to demonstrate glycogen storage in vascular smooth muscle fibres (black arrowheads). Original magnification: $630 \times$

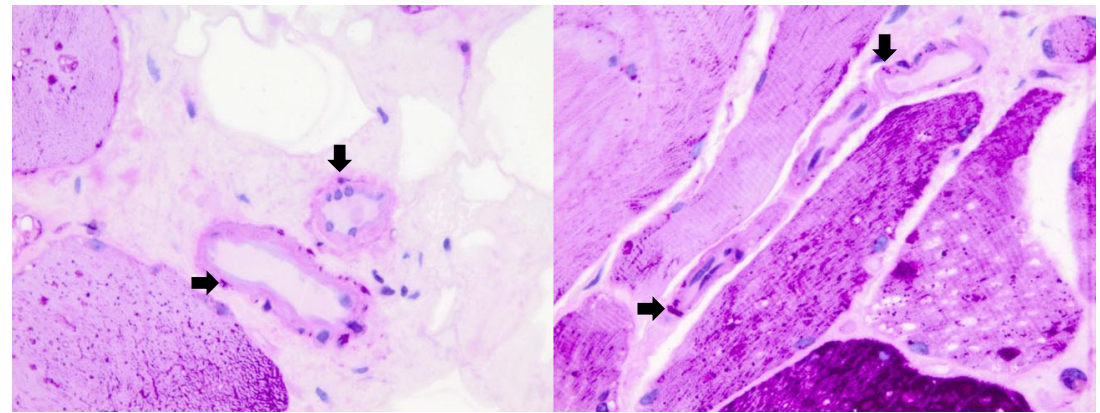


artery with age and with other cardiovascular risk factors such as increased body mass index and heart rate (Paini et al 2006). These findings may have been paralleled by our own finding in most of the Pompe patients whose blood pressure and heart rate were increased, who also had greater aortic stiffness without decreased distensibility.

A strength of our study is that we used cfPWV, which is currently the gold standard for measuring aortic stiffness directly and non-invasively in a relatively large group of Pompe patients. A previous study that found increased aortic stiffness in a small group of patients with Pompe disease used transthoracic echocardiography, an indirect way of measuring aortic stiffness (Nemes et al 2007).

Our study has some limitations. First, due to physical or temporary technical problems, measurements of aortic stiffness were not available for all subjects. However, as most of the patient data that were missing in the group of Pompe patients were those for the elderly and more severely affected patients, this would probably have led to an underestimation of the real difference in aortic stiffness between Pompe patients and healthy volunteers. Second, as this was a crosssectional study, we do not know about the progression of aortic stiffness over time, or about the effects of ERT on aortic stiffness. Additionally, rather than measuring glucose or cholesterol in blood samples, we used a questionnaire to establish whether patients had a history of hypercholesterolemia or diabetes mellitus.

In conclusion, patients with non-classic Pompe disease have increased aortic stiffness and blood pressure. To reduce the potential risk of cardiovascular diseases, we recommend that blood pressure and other common cardiovascular risk factors are monitored closely. Prospective studies should investigate whether ERT reduces aortic stiffness, or whether early treatment even prevents it.

\begin{abstract}
Acknowledgments As well as thanking patients and volunteers for participating in this study, we would like to thank Ms Evelien de Jager for her support duties, Dr Rob Verdijk for his expert judgment of the muscle biopsies and David Alexander for critically reviewing the manuscript. Research on Pompe disease at Erasmus MC is funded by the Erasmus MC Revolving Fund [project number 1054, NAMEvdB]; the European Union 7th Framework Programme "Euclyd - a European Consortium for Lysosomal Storage Diseases" [health F2/2008 grant number 201678]; ZonMw - Netherlands organization for health research and development [grant number 152001005]; and the Prinses Beatrix Spierfonds [project number OP07-08]. Since August 2004, ATvdP has provided consulting services for Genzyme Corp, Cambridge, MA, USA, under an agreement between Genzyme Corp and Erasmus MC University Medical Center, Rotterdam, The Netherlands.
\end{abstract}

Conflict of interest Ans van der Ploeg has provided consulting services for Genzyme Corporation since August 2004. Stephan Wens, Esther Kuperus, Francesco Mattace-Raso, Michelle Kruijshaar, Esther Brusse, Kees van Montfort, Marjan Scheltens- de Boer, Eric Sijbrands and Pieter van Doorn declare that they have no conflict of interest.
Open Access This article is distributed under the terms of the Creative Commons Attribution License which permits any use, distribution, and reproduction in any medium, provided the original author(s) and the source are credited.

\section{References}

Balkestein EJ, van Aggel-Leijssen DP, van Baak MA, Struijker-Boudier HA, Van Bortel LM (1999) The effect of weight loss with or without exercise training on large artery compliance in healthy obese men. $\mathrm{J}$ Hypertens 17(12 Pt 2):1831-1835

Braunsdorf WE (1987) Fusiform aneurysm of basilar artery and ectatic internal carotid arteries associated with glycogenosis type 2 (Pompe's disease). Neurosurgery 21(5):748-749

Collin C, Briet M, Tran TC et al (2012) Long-term changes in arterial structure and function and left ventricular geometry after enzyme replacement therapy in patients affected with Fabry disease. Eur J Prev Cardiol 19(1):43-54

DeLoach SS, Townsend RR (2008) Vascular stiffness: its measurement and significance for epidemiologic and outcome studies. Clin J Am Soc Nephrol 3(1):184-192

El-Gharbawy AH, Bhat G, Murillo JE et al (2011) Expanding the clinical spectrum of late-onset Pompe disease: dilated arteriopathy involving the thoracic aorta, a novel vascular phenotype uncovered. Mol Genet Metab 103(4):362-366

Girerd X, Giannattasio C, Moulin C, Safar M, Mancia G, Laurent S (1998) Regression of radial artery wall hypertrophy and improvement of carotid artery compliance after long-term antihypertensive treatment in elderly patients. J Am Coll Cardiol 31(5):1064-1073

Güngör D, Kruijshaar ME, Plug I et al (2013) Impact of enzyme replacement therapy on survival in adults with Pompe disease: results from a prospective international observational study. Orphanet J Rare Dis $8(1): 49$

Hirschhorn, R. (2001) Glycogen storage disease type II: acid alphaglucosidase (Acid Maltase) deficiency. The metabolic and molecular bases of inherited disease. In: Scriver CR, Sly WS, Valle D, New York, Mc Graw-Hill p3389-3420.

Hoeks AP, Brands PJ, Reneman RS (1992) Assessment of the arterial distension waveform using Doppler signal processing. J Hypertens Suppl 10(6):S19-S22

Huijben AM, Mattace-Raso FU, Deinum J, Lenders J, van den Meiracker AH (2012) Aortic augmentation index and pulse wave velocity in response to head-up tilting: effect of autonomic failure. J Hypertens 30(2):307-314

Kingwell BA, Berry KL, Cameron JD, Jennings GL, Dart AM (1997) Arterial compliance increases after moderate-intensity cycling. Am J Physiol 273(5 Pt 2):H2186-H2191

Kobayashi H, Shimada Y, Ikegami M et al (2010) Prognostic factors for the late onset Pompe disease with enzyme replacement therapy: from our experience of 4 cases including an autopsy case. Mol Genet Metab 100(1):14-19

Laforet P, Petiot P, Nicolino M et al (2008) Dilative arteriopathy and basilar artery dolichoectasia complicating late-onset Pompe disease. Neurology 70(22):2063-2066

Laurent S, Cockcroft J, Van Bortel L et al (2006) Expert consensus document on arterial stiffness: methodological issues and clinical applications. Eur Heart J 27(21):2588-2605

Mancia GR, Fagard K, Narkiewicz et al (2013) "2013 ESH/ESC Guidelines for the management of arterial hypertension.” Blood Press.

Mattace-Raso FU, van der Cammen TJ, Hofman A et al (2006) Arterial stiffness and risk of coronary heart disease and stroke: the Rotterdam Study. Circulation 113(5):657-663 
Nemes A, Soliman OI, Geleijnse ML et al (2007) Increased aortic stiffness in glycogenosis type 2 (Pompe's disease). Int J Cardiol 120(1):138-141

Nemes A, Timmermans RG, Wilson JH et al (2008) The mild form of mucopolysaccharidosis type I (Scheie syndrome) is associated with increased ascending aortic stiffness. Heart Vessels 23(2):108-111

Paini A, Boutouyrie P, Calvet D, Tropeano AI, Laloux B, Laurent S (2006) Carotid and aortic stiffness: determinants of discrepancies. Hypertension 47(3):371-376

Sacconi S, Bocquet JD, Chanalet S, Tanant V, Salviati L, Desnuelle C (2010) Abnormalities of cerebral arteries are frequent in patients with late-onset Pompe disease. J Neurol 257(10):1730-1733

Soliman OI, van der Beek NA, van Doorn PA et al (2008) Cardiac involvement in adults with Pompe disease. J Intern Med 264(4): 333-339

Thurberg BL, Lynch Maloney C, Vaccaro C et al (2006) Characterization of pre- and post-treatment pathology after enzyme replacement therapy for Pompe disease. Lab Invest 86(12):1208-1220

Van Bortel LM, Laurent S, Boutouyrie P et al (2012) Expert consensus document on the measurement of aortic stiffness in daily practice using carotid-femoral pulse wave velocity. J Hypertens 30(3): $445-448$ van den Hout HM, Hop W, van Diggelen OP et al (2003) The natural course of infantile Pompe's disease: 20 original cases compared with 133 cases from the literature. Pediatrics 112(2):332-340

van der Beek NA, de Vries JM, Hagemans ML et al (2012) Clinical features and predictors for disease natural progression in adults with Pompe disease: a nationwide prospective observational study. Orphanet J Rare Dis 7:88

van der Ploeg AT, Reuser AJ (2008) Pompe's disease. Lancet 372(9646): $1342-1353$

van Dijk SC, Smulders YM, Enneman AW et al (2013) Homocysteine level is associated with aortic stiffness in elderly: cross-sectional results from the B-PROOF study. J Hypertens 31(5):952-959

Verbeke F, Van Biesen W, Honkanen E et al (2011) Prognostic value of aortic stiffness and calcification for cardiovascular events and mortality in dialysis patients: outcome of the calcification outcome in renal disease (CORD) study. Clin J Am Soc Nephrol 6(1):153-159

Winkel LP, Kamphoven JH, van den Hout HJ et al (2003) Morphological changes in muscle tissue of patients with infantile Pompe's disease receiving enzyme replacement therapy. Muscle Nerve 27(6):743-751

Winkel LP, Hagemans ML, van Doorn PA et al (2005) The natural course of non-classic Pompe's disease; a review of 225 published cases. J Neurol 252(8):875-884 